\title{
EXPLOITATION OF ESA AND NASA HERITAGE REMOTE SENSING DATA FOR MONITORING THE HEAT ISLAND EVOLUTION IN CHENNAI WITH THE GOOGLE EARTH ENGINE
}

\author{
Francesca Cecinati, Donato Amitrano, Lemia Benevides Leoncio, Elvis Walugendo, Raffaella Guida, \\ Pasquale Iervolino, Sukumar Natarajan
}

\author{
Department of Architecture and Civil Engineering, University of Bath, Bath, UK \\ Surrey Space Centre, University of Surrey, Guildford UK \\ Nuffield Research Placement, UK
}

\begin{abstract}
Abstract - The Urban Heat Island (UHI) effect is defined as an increase of the air and surface temperature inside a city compared to surrounding rural areas. This increment can be of several degrees, thus exposing populations to serious health risks, especially in hot developing countries, where the majority of the world's megacities is located. The UHI effect has been widely studied in the past with local methods employing field sensors. The use of satellites moved the analysis from local to city scale, but long-term investigations have been so far limited by storage and computational capacities. In this work, both ESA and NASA heritage data are used to study the temporal evolution (2003-2017) of the UHI of the city of Chennai, India. The Google Earth Engine is exploited to process the available large dataset in a reasonable time. Results show that the UHI of Chennai has grown over time and that its main drivers are the average temperature and the city expansion.
\end{abstract}

Index Terms - Heritage data, MODIS, Urban Heat Island, Google Earth Engine

\section{INTRODUCTION}

In $2050,75 \%$ of the world population will live in cities [1]. City expansion is likely to strengthen the urban heat island (UHI) effect. This term indicates an increase of the surface and air temperature of an urban area compared to surrounding suburban and rural areas [2]. The UHI is due to the introduction of artificial materials that change the radiative, thermal, moisture conditions and emissivity of surfaces [3] and is cause of severe heat stress for the populations. The increase of temperature affects both the air in the urban boundary layer (Atmospheric Urban Heat Island - AUHI) and the urban surfaces (Surface Urban Heat Island - SUHI) [4]. In general, the UHI intensity depends on both environmental/climatic [5] and intrinsic urban factors like city shape, density, and extension [6].
Past work on UHI at city scale widely used temperature from ground stations, situated in urban and suburban areas [7]. Since the $90 \mathrm{~s}$, the increasing availability of satellite acquisitions moved the study of SUHI toward the exploitation satellite technologies [8]. In particular, starting from 2000, the NASA Moderate-Resolution Imaging Spectroradiometer (MODIS) sensor has been used to investigate the UHI effect. MODIS Land Surface Temperature (LST) products are available at $1 \mathrm{~km}$ resolution, twice per day and reach an accuracy of $1 \mathrm{~K}$ or better [9]. However, this large dataset, has not been fully exploited in the past due to technological limitations. In this work, the temporal evolution of the UHI of the city of Chennai, India (which represents an example of a large expanding city in a hot developing country), is investigated using all the available cloud-free daily MODIS LST acquisitions in the time frame 2003-2017. Temperature data are integrated with temporal urban area maps generated from both heritage and actual data delivered by the European Space Agency (ESA) Synthetic Aperture Radar (SAR) sensors ERS-1/2, ENVISAT, and Sentinel-1. Finally, UHI data are correlated with some environmental/climatic and urban variables, like the average temperature, the city size, the presence of vegetation, and the influence of El Niño, to find its main drivers.

The available dataset has been processed by exploiting the computational power offered The Google Earth Engine (GEE). It is a cloud platform developed by the private company Google, freely accessible for research purposes. It offers a developing environment with online access to petabytes of satellite and geographic data, access to Google computational capacity, and a set of specialized libraries for the optimal handling of big geospatial data [10]. The presented work has been supported by the work of highschool students doing a Nuffield Research Placement, therefore a simplified methodology has been used. 


\section{METHODOLOGY}

The main objectives of this work can be summarized as follows:

1. Identify the evolution of the Chennai urban area using ESA SAR images, producing a multitemporal urban map;

2. Use the GEE to combine MODIS LST daily products (MOD11A2.006) and the multi-temporal urban map to estimate the evolution of the SUHI;

3. Understand what the main drivers for the SUHI evolution are through a regression.

To this end, the methodology described by the flowchart represented in Figure 1 has been conceived. It draws from both ESA and NASA archives. ESA SAR data acquired by ERS-1/2, ENVISAT, and Sentinel-1 sensors are used in a multisensory fusion environment to retrieve the temporal evolution of the Chennai urban agglomerate. MODIS LST products are directly ingested in the GEE platform, which hosts the assimilation of SAR-derived data into the UHI estimation model and the regression analysis aiming at establish its main drivers.

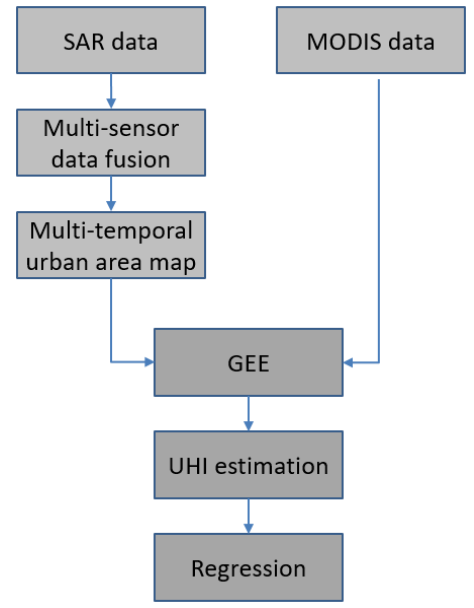

Fig. 1. Implemented workflow for the estimation of the UHI and the understanding of its main drivers

\section{CHENNAI CITY EXPANSION}

Long-term monitoring of the urban area is implemented by using SAR acquisitions of different sensors (ERS-1/2, ENVISAT, Sentinel-1) spanning from 1992 to 2017. Images were combined in bi-temporal multisensory colorcomposites according to the guidelines illustrated in [11] and the urban area extracted using dictionary-based semantic clustering principles, as discussed in [12]. The processing output, displayed in Figure 2, is a classified temporal urban map in which each class represents the state of the city for a given year.

\section{UHI ESTIMATION}

MODIS LST products are available only since 2000 . The first available urban map after 2000 is in 2003 . Therefore, the time frame $2003-2017$ was considered in this study for the investigation of the evolution of Chennai's SUHI.

The objective of this work is to understand how the SUHI has evolved over the years, regardless of the daily and seasonal oscillation. For this reason, the average LST, using all the available cloud-free MODIS LST acquisitions for each year corresponding to an available urban map, was computed. The average is calculated on a window of 3 years centered in the one of interest in order to avoid excessive inter-annual variability. The GEE platform allows for the handling and processing of this huge amount of data (about 570 MODIS images images overall) in a few minutes.

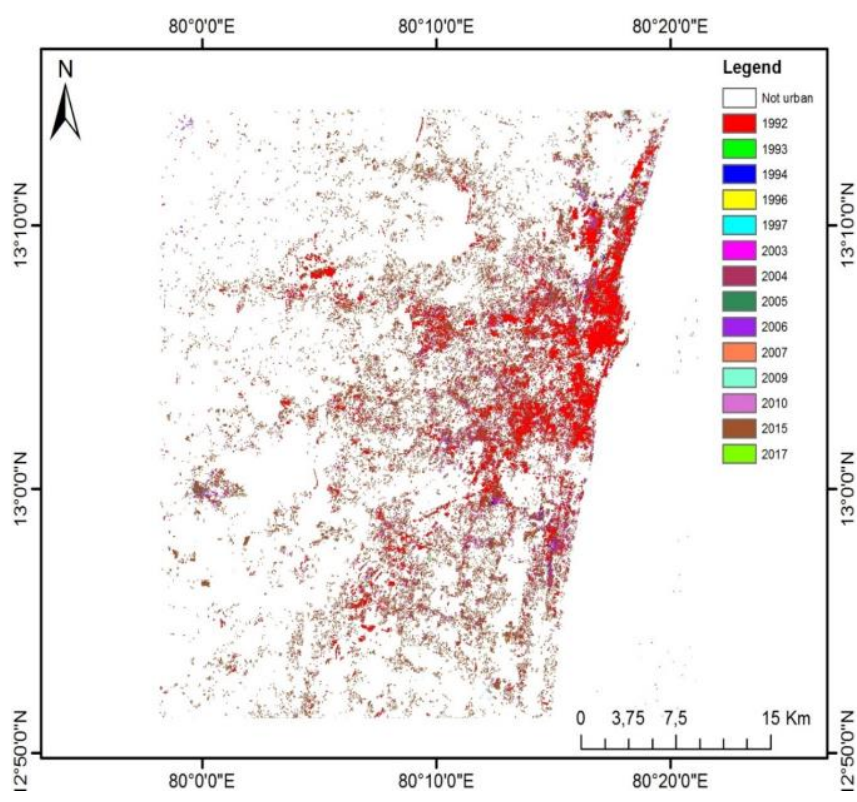

Fig. 2. Temporal urban area map of Chennai (India). Each color corresponds to the state of the city for a given year, starting from 1992 up to 2017.

Each average LST map is then segmented into urban/not urban classes according to the available SAR-derived map, ingested into the GEE. In Figure 3, an example relevant to the year 2017 is shown. In this picture, the left panel shows the average LST for pixels classified as urban. The right panel shows the average LST for pixels classified as not urban. Finally, the SUHI is calculated as follows

$$
\mathrm{SUHI}=\langle\mathrm{LST}\rangle_{\text {urban }}-\langle\mathrm{LST}\rangle_{\text {not-urban }}
$$

where the symbol $\langle *\rangle$ stands for the temporal mean operator and $L S T_{\text {urban }}$ and $L S T_{\text {not-urban }}$ for the LST measured by MODIS for urban and not urban pixels, respectively. This operation is repeated for all the available urban maps. The (linearly interpolated) estimated average SUHI is 

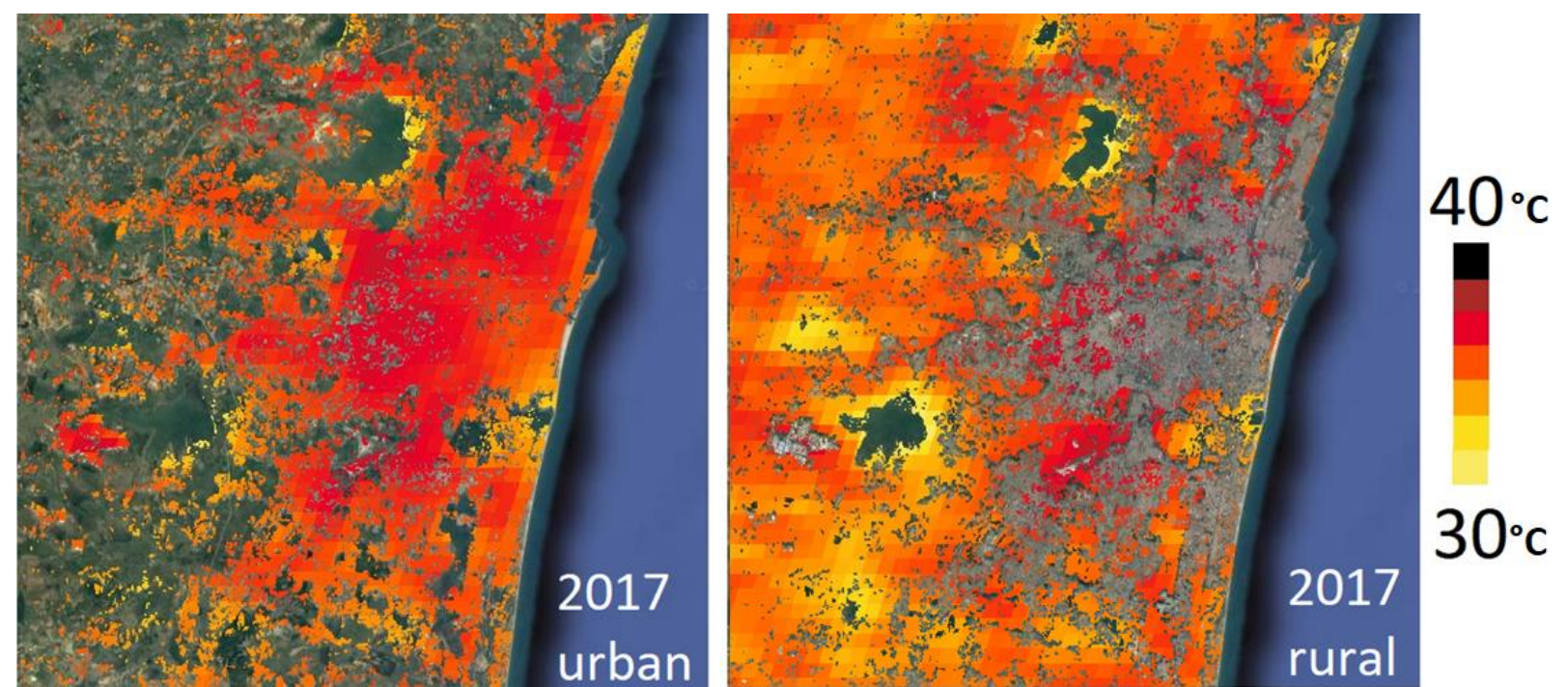

Fig. 3. Average LST distribution for the year 2017 in the Chennai area according to the SAR-derived urban/not urban segmentation.

represented by the black curve in the graphs depicted in Figure 4.

\section{UHI REGRESSION MODEL}

After calculating the average SUHI for the over the time, the factor most affecting its evolution were investigated. In particular, the following factors were considered:

- Average temperature $T_{\text {mean }}$ over the temporal window. Rural temperature is considered to avoid the UHI effect;

- $\quad$ City expansion, quantified by the maximum radius $R$ around the city center where the urban density is at least $30 \%$;

- Vegetation, quantified by the Normalized Difference Vegetation Index (NDVI) calculated from Landsat 7 images averaged in the same time window used to estimate the average SUHI;

- El Niño Southern Oscillation, quantified by the average Southern Oscillation Index (SOI) over the aforementioned temporal window. The SOI is defined as the annual standardised monthly mean sea level pressure anomalies at Tahiti, French Polynesia and Darwin, Australia [13].

The temporal evolution of the explanatory factors is shown in Figure 5, together with the temporal evolution of the SUHI. A linear regression is performed between the SUHI time series and each of the factors. Results are reported in Table 1 . The average temperature showed a strong inverse correlation with the SUHI and a strong direct correlation with the city expansion. The SOI showed a significant, but not strong direct correlation with the SUHI. The NDVI did not show a significant correlation with the SUHI evolution. A multiple linear regression between the SUHI and the three most significant explanatory factors
( $T_{\text {mean }}, R$, and SOI) and between the SUHI and the most significant two explanatory factors $\left(T_{\text {mean }}\right.$ and $\left.R\right)$ has been also implemented. Results are reported in Table 2. They show that the SOI does not add a noticeable contribution explaining the SUHI variability.

Table 1. Pearson's correlation $P$ and significance at $95 \%$ confidence $S_{95 \%}$ for the regression between the estimated average SUHI and the four considered explanatory factors.

\begin{tabular}{ccc}
\hline & $P$ & $S_{95 \%}$ \\
\hline \hline$T_{\text {mean }}$ & -0.710 & Yes \\
$S O I$ & 0.682 & Yes \\
$R$ & 0.842 & Yes \\
$N D V I$ & -0.342 & No \\
\hline \hline
\end{tabular}

Table 2. Result of the multiple linear regression between the average SUHI and the considered explanatory factors.

\begin{tabular}{ccc}
\hline \hline & $P$ & $S_{95 \%}$ \\
\hline \hline$T_{\text {mean }}, R$ & -0.710 & Yes \\
$T_{\text {mean }}, R, S O I$ & 0.682 & Yes \\
\hline
\end{tabular}

\section{CONCLUSIONS}

Urban heat islands represent a serious consequence of increasing urbanization and is expected to be furtherly exacerbated by the increasing temperatures our planet is experiencing. In this work, heritage remote sensing data were exploited for estimating the temporal evolution of the Urban Heat Island of the city of Chennai (India) using the Google Earth Engine. Results show an increasing trend of the average surface urban heat island effect. It is well explained by the city growth and by the average temperature, for which strong positive and negative correlation have been found. The Google Earth Engine proved to be a precious resource to process long time series, 

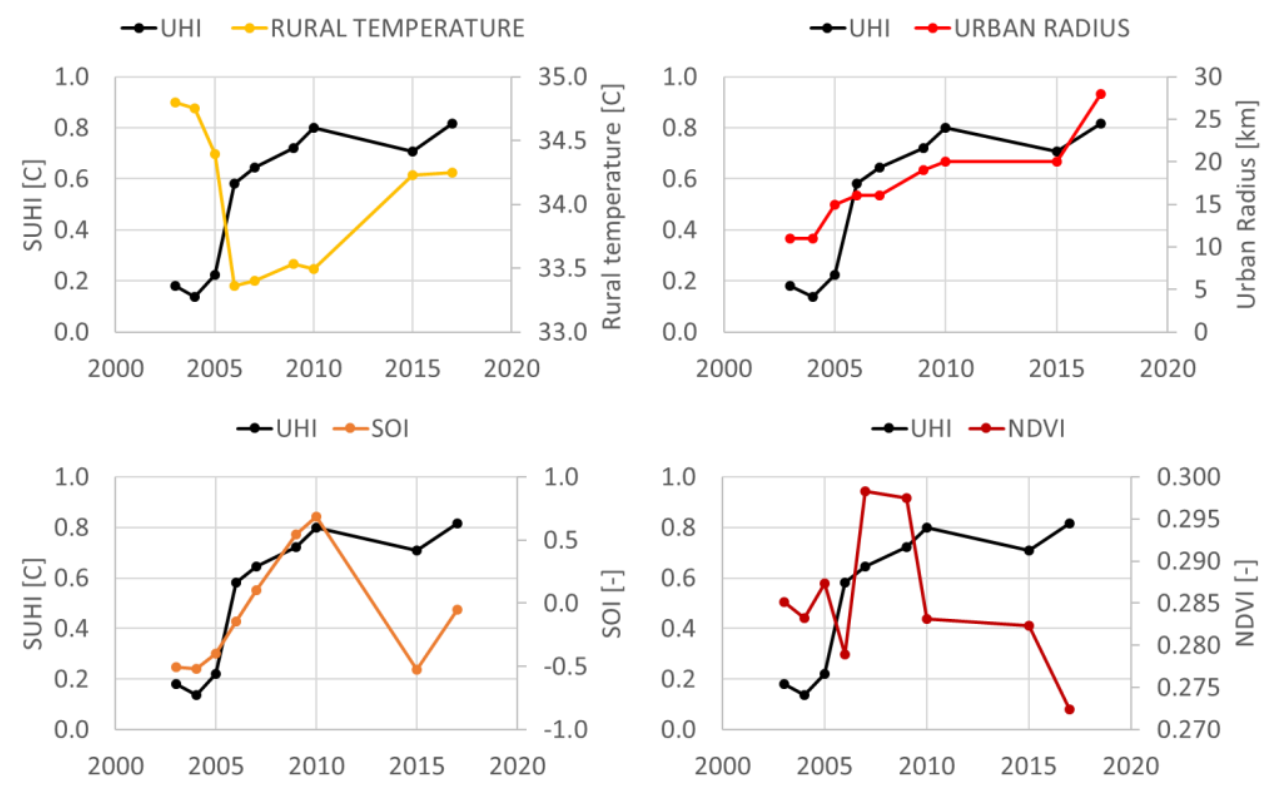

Fig. 4. Temporal evolution of the estimated SUHI and of the considered explanatory factors, i.e. average temperature, urban radius, southern oscillation index, and vegetation.

allowing for the development of efficient algorithms that are re-applicable to other case studies with negligible computational time and without necessity of huge storage units thanks to the possibility to move the processing in a cloud environment. Furthermore, its simplicity allows nonexpert users, like high-school students from the Nuffield Research Placement, to conduct meaningful remote sensing research.

\section{ACKNOWLEDGMENTS}

The research is funded by the EPSRC project Zero Peak Energy Building Design for India (EP/R008612/1) and by the GB Sasakawa Foundation grant No. 5544. The authors thank the European Space Agency for providing historical ASAR and ERS data under the aegis of the project "Long term satellite observations for urban areas and water resources monitoring", and the Nuffield Research Foundation for supporting two Research Placements.

\section{REFERENCES}

[1] UNDESA, "World Population Prospects: The 2014 Revision, Highlights," New York, 2014.

[2] S. Peng et al., "Surface urban heat island across 419 global big cities," Environ. Sci. Technol., vol. 46, no. 2, pp. 696-703, 2012.

[3] J. juan Li, X. rong X. jun Wang, X. rong X. jun Wang, W. chun Ma, and H. Zhang, "Remote sensing evaluation of urban heat island and its spatial pattern of the Shanghai metropolitan area, China," Ecol. Complex., vol. 6, no. 4, pp. 413-420, 2009.

[4] T. R. Oke, "The energetic basis of the urban heat island," Q. J. R. Metereological Soc., vol. 108, pp. 1-24, 1982.
[5] J. Unger, Z. Sumeghy, and J. Zoboki, "Temperature cross-section features in an urban area," Atmos. Res., vol. 58, pp. 117-127, 2001.

[6] B. Zhou, D. Rybski, and J. P. Kropp, "The role of city size and urban form in the surface urban heat island," Sci. Rep., vol. 7, no. 1, pp. 1-9, 2017.

[7] P. I. Figuerola and N. A. Mazzeo, "Urban-rural temperature differences in Buenos Aires," Int. J. Climatol., vol. 18, pp. 1709-1723, 1998.

[8] A. Rasul et al., "A Review on Remote Sensing of Urban Heat and Cool Islands," Land, vol. 6, no. 2, p. 38, 2017.

[9] Z. Wan, Y. Zhang, Q. Zhang, and Z. liang Li, "Validation of the land-surface temperature products retrieved from terra moderate resolution imaging spectroradiometer data," Remote Sens. Environ., vol. 83, no. 1-2, pp. 163-180, 2002.

[10] N. Gorelick, M. Hancher, M. Dixon, S. Ilyushchenko, D. Thau, and R. Moore, "Google Earth Engine: Planetaryscale geospatial analysis for everyone," Remote Sens. Environ., vol. 202, pp. 18-27, 2017.

[11] D. Amitrano, G. Di Martino, A. Iodice, D. Riccio, and G. Ruello, “A New Framework for SAR Multitemporal Data RGB Representation: Rationale and Products," IEEE Trans. Geosci. Remote Sens., vol. 53, no. 1, pp. 117-133, 2015.

[12] D. Amitrano, F. Cecinati, G. Di Martino, A. Iodice, P.P. Mathieu, D. Riccio, and G. Ruello, "Feature Extraction from Multitemporal SAR Images Using Selforganizing Map Clustering and Object-Based Image Analysis," IEEE J. Sel. Topics Appl. Earth Observ., vol. 11, no. 5, pp. 1556-1570. [13] NOAA, Southern Oscillation Index (SOI), https://www.ncdc.noaa.gov/teleconnections/enso/indicators/s oi/ accessed on 03/12/2018. 